\title{
Casimir corrections in the bound state soliton model
}

\author{
Norberto N. SCOCCOLA ${ }^{a}$ and Hans WALLISER ${ }^{b}$ \\ a Physics Department, Comisión Nacional de Energía Atómica, \\ Av.Libertador 8250, (1429) Buenos Aires, Argentina. \\ ${ }^{b}$ Departamento de Física, Universidade de Coimbra, \\ P-3000 Coimbra, Portugal. \\ (May 1998)
}

\begin{abstract}
We consider the one-loop corrections to the $S U(3)$ skyrmion mass within the bound state soliton approach. We show that the standard $S U(3)$ renormalization scheme is not appropiate within this framework and propose to use an alternative one based on $S U(2)$. For physical meson masses and decay constants the resulting Casimir correction turns out to be rather scale-independent and leads to an acceptable estimate of the nucleon mass.
\end{abstract}

PACS number(s): 12.39.Fe, 13.39.Dc, 12.40.Yx

\section{INTRODUCTION}

It is well-known that the nucleon mass as calculated within the $S U(2)$ Skyrme model at tree level comes out to be roughly $50 \%$ too high, when empirical values are used for the model parameters. For a long period, this was considered to be one of the main drawbacks of the model. It was only at the beginning of this decade that it was realized [1] that the proper inclusion of one-loop effects could reduce the skyrmion mass down to a value which is in reasonable agreement with the empirical nucleon mass. Since then the role of these effects on various nucleon properties have been investigated. Quite recently [2] it has been shown that, with the exception of some axial properties, pionic loop corrections tend to bring all the adiabatic quantities close to their experimental values. For those axial properties there seems to be some difficulties with the $1 / N_{c}$ expansion. Still, simple estimates of the next-to-next-to-leading order corrections suggest that they could already remedy the defects that show up if one includes only adiabatic one-loop contributions. Given the importance of the loop corrections in several $S U(2)$ skyrmion properties, it is clear that their role within the $S U$ (3) Skyrme model is worth to be investigated. Unfortunately, it is not clear whether the formalism used in the non-strange sector can be easily extended to the $S U(3)$ model. The basic problem is the presence of a rather large symmetry breaking term which makes the standard renormalization scheme rather unreliable. In fact, as a consequence of such term two alternative approaches to the $S U(3)$ soliton models have been developed. One is based on the straighforward extension of the $S U(2)$ collective coordinate quantization to the $S U(3)$ flavor group [3. Due to the existence of additional rotational modes the predictions for the tree level baryon masses are even worse than those in the $S U(2)$ Skyrme model, with values which typically lie around $2 \mathrm{GeV}$. The other approach to strange skyrmions [A. 5] assumes that the kaon mass is large enough to allow only for small amplitude fluctuations along the strangeness direction. Thus, hyperons appear as soliton-kaon bound systems. Since in this approach there are no additional rotational modes one might expect that pion loops already account for the necessary Casimir corrections. Then, one has to care about the role of the kaon and eta loops which, as we will show later, in the standard renormalization scheme diverge in the large mass limit. As we see, even for the Casimir energies the situation in the $S U(3)$ Skyrme model is unclear and has to be carefully studied. As a first step in such investigations one of us recently reported [6] the results concerning one-loop corrections in the flavor symmetric limit $\Delta m=m_{K}-m_{\pi}=0$. Such calculations have been done using the $S U(3)$ collective quantization scheme and show that the strangeness degrees of freedom tend to push the nucleon mass even further down. Namely, for the $S U(3)$ case in the flavor symmetric limit the nucleon mass is $770 \mathrm{MeV}$ to be compared with the $S U(2)$ prediction $1020 \mathrm{MeV}$. Since these two cases correspond to $\Delta m=0$ and $\Delta m \rightarrow \infty$ respectively, for empirical values of $\Delta m$ the predicted mass is expected to lie between these two limiting values. However, the methods used in Ref. [6] cannot be easily used for finite $m_{K} \neq m_{\pi}$. In this paper we introduce an alternative way to evaluate the Casimir correction which is suitable for large values of $\Delta m$. This will allow us to compute the one-loop corrections in the bound state approach.

This article is organized as follows. In Sec. 2 we briefly derive the basic formulae needed to evaluate the one loop corrections to the skyrmion mass in the bound state approach. In Sec. 3 we discuss the possible 
ways to renormalize these corrections. In Sec. 4, we present the numerical results corresponding to these different renormalization schemes. Finally, Sec. 5 contains some discussions and conclusions.

\section{FORMULATION}

This investigation is based on the standard chiral $S U(3)$ lagrangian [7]

$$
\begin{aligned}
\mathcal{L} & =\frac{F^{2}}{4} \operatorname{tr}\left[\partial_{\mu} U \partial^{\mu} U^{\dagger}+M\left(U+U^{\dagger}\right)\right] \\
& +\left(L_{1}+L_{2}+\frac{1}{2} L_{3}\right)\left(\operatorname{tr} \partial_{\mu} U \partial^{\mu} U^{\dagger}\right)^{2}+\frac{1}{2} L_{2} \operatorname{tr}\left(\left[U^{\dagger} \partial_{\mu} U, U^{\dagger} \partial_{\nu} U\right]\right)^{2} \\
& +\left(L_{3}+3 L_{2}\right)\left[\operatorname{tr} \partial_{\mu} U \partial^{\mu} U^{\dagger} \partial_{\nu} U \partial^{\nu} U^{\dagger}-\frac{1}{2}\left(\operatorname{tr} \partial_{\mu} U \partial^{\mu} U^{\dagger}\right)^{2}\right] \\
& +L_{4} \operatorname{tr} M\left(U+U^{\dagger}\right) \operatorname{tr} \partial_{\mu} U \partial^{\mu} U^{\dagger}+L_{5} \operatorname{tr}\left(U M+M U^{\dagger}\right) \partial_{\mu} U \partial^{\mu} U^{\dagger} \\
& +L_{6}\left(\operatorname{tr} M\left(U+U^{\dagger}\right)\right)^{2}+L_{7}\left(\operatorname{tr} M\left(U-U^{\dagger}\right)\right)^{2} \\
& +L_{8} \operatorname{tr}\left(M U M U+M U^{\dagger} M U^{\dagger}\right) \\
& \equiv \frac{F^{2}}{4} \operatorname{tr}\left[\partial_{\mu} U \partial^{\mu} U^{\dagger}+M\left(U+U^{\dagger}\right)\right]+\sum_{i=1}^{8} L_{i} \mathcal{L}_{i}^{(4)} .
\end{aligned}
$$

expressed in terms of the matrix $U$ which contains the dynamical fields and the mass matrix

$$
M=\left(\begin{array}{ccc}
m_{\pi}^{2} & & \\
& m_{\pi}^{2} & \\
& & 2 m_{K}^{2}-m_{\pi}^{2}
\end{array}\right) .
$$

In (17) we listed the familiar non-linear sigma $(\mathrm{N} \ell \sigma)$ model of chiral order $\mathrm{ChO} 2$ and eight terms of $\mathrm{ChO} 4$ which are relevant in the soliton sector without external fields. The Wess-Zumino-Witten (WZW) term is included although not explicitly denoted. After renormalization, the low energy constants (LECs) $L_{i}(\mu)$ become dependent on the chiral scale $\mu$ and the choice $\mu \simeq m_{\varrho}=770 \mathrm{MeV}$ should provide the lagrangian in leading order $N_{c}[8]$. Therefore, at this scale

$$
2 L_{1}\left(m_{\varrho}\right)-L_{2}\left(m_{\varrho}\right)=L_{4}\left(m_{\varrho}\right)=L_{6}\left(m_{\varrho}\right)=0
$$

because these combinations are subleading in $N_{c}$ [7]. We postpone the choice of the remaining LECs to the following section where we discuss the renormalization schemes.

The starting point of the bound state approach (BSA) is the hedgehog solution rotating in $S U(2)$ only,

$$
U_{0}=\left(\begin{array}{ll}
A e^{i \boldsymbol{\tau} \cdot \hat{\boldsymbol{r}}_{F(r)}} A^{\dagger} & \\
& 1
\end{array}\right) \quad A \in S U(2) .
$$

Because the rotation matrix $A$ commutes with the mass matrix (2) it does not appear in the adiabatic approximation where time derivatives on the collective coordinates are neglected. For the description of strange hyperons and also for the 1-loop calculation considered here, fluctuations $\eta_{a}$ are introduced through the ansatz [4]

$$
U=\sqrt{U_{0}} e^{i \lambda_{a} \eta_{a} / F} \sqrt{U_{0}}, \quad a=1, \ldots, 8 .
$$

Of particular interest are kaonic $(a=4, \ldots, 7)$ and eta $(a=8)$ fluctuations; pionic fluctuations have already been calculated in Ref. [2]. The corresponding equations of motion (e.o.m.) may be generically written as

$$
h_{a b}^{2} \eta_{b}+i \omega \frac{N_{c} B_{0}}{\sqrt{3} f_{\pi}^{2}} f_{8 a b} \eta_{b}=\omega^{2} n_{a b}^{2} \eta_{b}
$$

where $h_{a b}^{2}$ is a differential operator, $n_{a b}^{2}$ the metric and the linear term in the eigenenergy $\omega$ accounts for the WZW term that appears in the kaonic case. The partial wave projected kaonic e.o.m. is explicitely given 
e.g. in Ref. [9]. In general, these equations have to be solved for the phase-shifts. Since they decouple for the different meson species into partial waves characterized by phonon spin $L$ and parity, the pionic, kaonic and eta phase-shifts may be summed up separately over the various channels $(L, c)$

$$
\delta^{x}(p)=\sum_{L, c}(2 L+1) \delta_{L, c}^{x}(p) \quad x=\pi, K, \eta .
$$

The ultra-violet divergencies contained in the Casimir energy are related to the high momentum behaviour of these phaseshifts

$$
\delta^{x}(p) \stackrel{p \rightarrow \infty}{\longrightarrow} a_{0}^{x} p^{3}+a_{1}^{x} p+\frac{a_{2}^{x}}{p}+\cdots
$$

with expansion coefficients $a_{0}^{x}, a_{1}^{x}, a_{2}^{x}$ known analytically for the $\mathrm{N} \ell \sigma$ model (the explicitly denoted terms give rise to at least logarithmically divergent expressions). For the full model (11) the coefficients have to be determined numerically and the challenge is to calculate the phase-shifts with great precision up to $p_{\max } \simeq 25 m_{\pi}$ where $L_{\max } \simeq 100$ partial waves are needed (for details see [2]). The 1-loop contribution is then given by [1, 2, 6, 6 ]

$$
\begin{aligned}
E_{\text {cas }}= & \frac{1}{2 \pi} \sum_{x}\left(-\int_{0}^{\infty} \frac{p d p}{\sqrt{p^{2}+m_{x}^{2}}}\left[\delta^{x}(p)-a_{0}^{x} p^{3}-a_{1}^{x} p-\frac{a_{2}^{x}}{p}\right]-m_{x} \delta^{x}(0)\right. \\
& \left.+\frac{3 m_{x}^{4} a_{0}^{x}}{16}\left(\frac{1}{6}+\ln \frac{m_{x}^{2}}{\mu^{2}}\right)-\frac{m_{x}^{2} a_{1}^{x}}{4} \ln \frac{m_{x}^{2}}{\mu^{2}}+\frac{a_{2}^{x}}{2}\left(1+\ln \frac{m_{x}^{2}}{\mu^{2}}\right)\right) \\
& +\Lambda(\mu) \sum_{x}\left[3 \pi m_{x}^{4} a_{0}^{x}-4 \pi m_{x}^{2} a_{1}^{x}+8 \pi a_{2}^{x}\right]+\frac{1}{2} \sum_{b} \omega_{b} .
\end{aligned}
$$

The last term in Eq. (9) represents the contribution coming from all possible bound states. The chiral scale $\mu$ is introduced to render the arguments in the logarithms dimensionless and the divergencies as $d \rightarrow 4$ reside in

$$
\Lambda(\mu)=\frac{\mu^{d-4}}{16 \pi^{2}}\left[\frac{1}{d-4}-\frac{1}{2}\left(\Gamma^{\prime}(1)+\ln (4 \pi)+1\right)\right] .
$$

In order to make sense out of these expressions they have to be properly renormalized. This issue is discussed in the next section.

\section{THE RENORMALIZATION SCHEMES}

There are various possibilities to renormalize the expression (9) which we are going to discuss subsequently.

\section{A. Standard $S U(3)$ renormalization scheme}

Noting that the $\mathrm{N} \ell \sigma$ model expansion coefficients obey the ChO4 relation

$$
\sum_{x}\left[3 \pi m_{x}^{4} a_{0}^{x}-4 \pi m_{x}^{2} a_{1}^{x}+8 \pi a_{2}^{x}\right]=\sum_{i=1}^{8} \Gamma_{i} \int d^{3} r \mathcal{L}_{i}^{(4)},
$$

the divergencies in (9) may be absorbed into a redefinition of the lagrangian's LECs

$$
L_{i}(\mu)=L_{i}-\Gamma_{i} \Lambda(\mu), \quad L_{i}(\mu)=L_{i}\left(m_{\varrho}\right)-\frac{\Gamma_{i}}{32 \pi^{2}} \ln \left(\frac{\mu^{2}}{m_{\varrho}^{2}}\right)
$$

which become scale-dependent. The $\Gamma_{i}$ 's are simple numerical factors given in Table 1 and coincide with those defined in [7]. $F^{2}$ and the mass matrix $M$ are not renormalized in that scheme which is identical to that used in $S U(3)$ chiral perturbation theory. 
The remaining piece in (9) is the finite Casimir energy for the three meson species

$$
\begin{aligned}
E_{\mathrm{cas}}^{x}(\mu)= & -\frac{1}{2 \pi} \int_{0}^{\infty} \frac{p d p}{\sqrt{p^{2}+m_{x}^{2}}}\left[\delta^{x}(p)-a_{0}^{x} p^{3}-a_{1}^{x} p-\frac{a_{2}^{x}}{p}\right]-\frac{m_{x}}{2 \pi} \delta^{x}(0) \\
& +\frac{3 m_{x}^{4} a_{0}^{x}}{32 \pi}\left(\frac{1}{6}+\ln \frac{m_{x}^{2}}{\mu^{2}}\right)-\frac{m_{x}^{2} a_{1}^{x}}{8 \pi} \ln \frac{m_{x}^{2}}{\mu^{2}}+\frac{a_{2}^{x}}{4 \pi}\left(1+\ln \frac{m_{x}^{2}}{\mu^{2}}\right)+\frac{1}{2} \sum_{b} \omega_{b}
\end{aligned}
$$

which has to be added to the static soliton mass calculated with the renormalized LECs (12)

$$
E_{\text {tree+1-loop }}=M_{\text {sol }}(\mu)+E_{\text {cas }}^{\pi}(\mu)+E_{\text {cas }}^{K}(\mu)+E_{\text {cas }}^{\eta}(\mu) .
$$

In order to obtain at scale $\mu=m_{\varrho}$ the same soliton as in the $S U(2)$ calculation [2] we fix $F=91.1 \mathrm{MeV}$ and the remaining LECs according to (3) and

$$
\begin{aligned}
& L_{2}\left(m_{\varrho}\right)=\frac{1}{16 e^{2}}, \quad L_{3}\left(m_{\varrho}\right)+3 L_{2}\left(m_{\varrho}\right)=0 \\
& L_{5}\left(m_{\varrho}\right)=2 L_{8}\left(m_{\varrho}\right)=-6 L_{7}\left(m_{\varrho}\right)=2.3 \cdot 10^{-3}
\end{aligned}
$$

which is in accordance with the standard values [7, 8 (within error bars) with one exception: $L_{2}$ has to be replaced by an effective Skyrme parameter $e=4.25$ (the standard value would correspond to $e \simeq 7$ ) in order to simulate the missing higher ChOs generated by vector mesons. A detailed justification of this choice used also in [6] is found in Ref. [2]. It should be mentioned that although the LECs are chosen such that many of the ChO4 terms in (11) vanish at scale $\mu=m_{\varrho}$ all these terms are switched on and do contribute when the scale is changed.

As it is obvious from Eq.(13), this renormalization scheme becomes increasingly unreliable as $m_{x}$ becomes closer or larger than the renormalization scale $\mu$. In such cases, an alternative scheme is needed.

\section{B. $S U(2)$ renormalization scheme}

For large kaon mass the troublesome terms are located in the kaon's and eta's Casimir energies. While the phase-shift integral in Eq.(13) behaves well, the extra terms multiplied with the chiral logs explode with increasing kaon mass. It is now possible to renormalize also these terms into the lagrangian

$$
\overline{\mathcal{L}}=\frac{\bar{F}^{2}}{4} \operatorname{tr}\left[\partial_{\mu} U \partial^{\mu} U^{\dagger}+\bar{M}\left(U+U^{\dagger}\right)\right]+\sum_{i=1}^{8} \bar{L}_{i} \mathcal{L}_{i}^{(4)} .
$$

with the $\bar{L}_{i}^{\prime}$ s given in Table 1 and

$$
\begin{aligned}
& \qquad \bar{F}^{2}=F^{2}\left(1-2 \mu_{K}\right)+m_{\pi}^{2} \nu_{K} \\
& \bar{F}^{2} \bar{m}_{x}^{2}=m_{x}^{2}\left[F^{2}\left(1-2 \mu_{K}-\frac{1}{3} \mu_{\eta}\right)+m_{\pi}^{2}\left(\nu_{K}+\frac{1}{9} \nu_{\eta}\right)-\left(2 m_{K}^{2}-m_{\pi}^{2}\right)\left(\frac{1}{6} \nu_{K}+\frac{1}{9} \nu_{\eta}\right)\right] \\
& \text { where } \quad \nu_{x}=\frac{1}{32 \pi^{2}}\left(1+\ln \frac{m_{x}^{2}}{\mu^{2}}\right), \quad \mu_{x}=\frac{m_{x}^{2}}{32 \pi^{2} F^{2}} \ln \frac{m_{x}^{2}}{\mu^{2}} .
\end{aligned}
$$

All these quantities become scale-dependent. For $\bar{F}$ and $\bar{m}_{x}$ this dependence may be read directly from their definition (17) and correspondingly for the $\bar{L}_{i}$

$$
\bar{L}_{i}(\mu)=\bar{L}_{i}\left(m_{\varrho}\right)-\frac{\bar{\Gamma}_{i}}{32 \pi^{2}} \ln \left(\frac{\mu^{2}}{m_{\varrho}^{2}}\right)
$$

with coefficients $\bar{\Gamma}_{i}$ obtained in $S U(2)[7]$ and listed in Table 1. However, in contrast to the standard $S U(3)$ coefficients, the bared LECs $\bar{L}_{i}$ do not depend on the kaon mass; in fact the kaon mass dependence of the $S U(3)$ LECs $L_{i}$ may be deduced from these relations.

In order to make the connection to $S U(2)$ more transparent we give the relation for the corresponding LECs $f, m$, and $\ell_{i}(i=1, \ldots, 4)$ explicitely [7] 


$$
\begin{aligned}
& f^{2}=\bar{F}^{2}+8\left(2 m_{K}^{2}-m_{\pi}^{2}\right) \bar{L}_{4} \\
& f^{2} m^{2}=\bar{m}_{\pi}^{2}\left[\bar{F}^{2}+16\left(2 m_{K}^{2}-m_{\pi}^{2}\right) \bar{L}_{6}\right] \\
& \ell_{1}=4 \bar{L}_{1}+2 \bar{L}_{3} \\
& \ell_{2}=4 \bar{L}_{2} \\
& \ell_{3}=-8 \bar{L}_{4}-4 \bar{L}_{5}+16 \bar{L}_{6}+8 \bar{L}_{8} \\
& \ell_{4}=8 \bar{L}_{4}+4 \bar{L}_{5} .
\end{aligned}
$$

It is readily checked that the $\ell_{i}$ have the correct scale-dependence 10$]$.

Let us turn back to the 1-loop calculation of the soliton energy. While the expression for the pionic Casimir energy is unaltered the kaonic and eta Casimir energies are now given exclusively by the phase-shift integral and the bound state contributions

$$
\begin{aligned}
\bar{E}_{\mathrm{cas}}^{x}(\mu)= & -\frac{1}{2 \pi} \int_{0}^{\infty} \frac{p d p}{\sqrt{p^{2}+m_{x}^{2}}}\left[\delta^{x}(p)-a_{0}^{x} p^{3}-a_{1}^{x} p-\frac{a_{2}^{x}}{p}\right]-\frac{m_{x}}{2 \pi} \delta^{x}(0) \\
& +\frac{1}{2} \sum_{b} \omega_{b}, \quad x=K, \eta
\end{aligned}
$$

without the troublesome extra terms. Therefore the total soliton energy in tree +1 -loop is given by

$$
\bar{E}_{\text {tree+1-loop }}=\bar{M}_{\text {sol }}(\mu)+E_{\text {cas }}^{\pi}(\mu)+\bar{E}_{\text {cas }}^{K}(\mu)+\bar{E}_{\text {cas }}^{\eta}(\mu) .
$$

Note that for kaons the last two terms in Eq.(20) exactly cancel out in the limit of infinite kaon mass. As a consequence the kaonic contribution remains finite even in that limit.

\section{NUMERICAL RESULTS}

The physical kaon and eta masses are somewhat smaller, although comparable, than the chosen scale $m_{\rho}$. In this sense, it is not a priori clear which renormalization scheme is more appropiate. We investigate this issue numerically. We consider first the $S U(3)$ renormalization scheme assuming that the meson masses contained in the mass matrix take their physical values. As a consequence of the choice (15) the pionic Casimir energy is the same as in the $S U(2)$ case where we found scale-independence of the soliton energy in tree +1 -loop over a wide region of chiral scales [2]. It should be mentioned that the scale-dependence of the LECs (12) differs slightly from that of the LECs used in $S U(2)$ but that does not affect the statement concerning the scale-independence of the soliton energy. Because the coupling of the eta to the soliton proceeds through the mass terms only, it is extremely weak: the Casimir energy of the eta amounts to $E_{\text {cas }}^{\eta}\left(m_{\rho}\right)=1.7 \mathrm{MeV}$ and can savely be omitted. Therefore in what follows we concentrate on the kaonic contribution to the Casimir energy $E_{\text {cas }}^{K}$. In this sector we get two doublets of bound states. For the model parameters we use, the S-wave doublet appears at $\omega_{S}=465 \mathrm{MeV}$ and the P-doublet at $\omega_{P}=256 \mathrm{MeV}$.

The kaonic Casimir energy is shown in Fig. 1 depending on the chiral scale $\mu$ (dashed line). For $\mu=$ $m_{\rho}=770 \mathrm{MeV}$ we obtain a positive contribution of $\simeq 140 \mathrm{MeV}$ which has to be added to the $S U(2)$ soliton energy in tree +1 -loop of $1020 \mathrm{MeV}$ in order to get the total nucleon mass. We observe a considerable scale-dependence of the kaonic Casimir energy which may have one of the following reasons: (i) there may be important terms missing in the lagrangian, (ii) the hedgehog rotating in $S U(2)$ as used in the BSA might be not an appropriate solution for physical kaon mass, or (iii) the employed renormalization scheme could be incompatible with that approach.

In order to shed some light onto this issue we are going to investigate the dependence on the kaon mass in more detail. Particularly, we know that the BSA should become accurate for large kaon masses. The dependence of the kaonic Casimir energy on the kaon mass is shown in Fig. 2 (dashed line). We observe a drastic dependence and, as expected, we notice that the kaonic Casimir energy does not tend to zero for large kaon masses as it should. This is of course no surprise because the experimental LECs (12) were evaluated for physical kaon mass and should be used only there. This study of the kaon mass dependence indicates that the $S U(2)$ renormalization scheme which allows the LECs to run smoothly into their $S U(2)$ values for large kaon masses might be more suitable for the BSA.

In the $S U(2)$ renormalization scheme we start by choosing at the scale $\mu=m_{\rho}$ the bared quantities in exactly the same way as the unbared ones above. This guarantees that the soliton mass and the pionic 
Casimir energy as well as their scale-dependences are exactly the same as in $S U(2)$. Note that this was not exactly true in the $S U(3)$ renormalization scheme where the LECs scaled in a slightly different way. We may again forget about the tiny eta Casimir energy $\left(\bar{E}_{\text {cas }}^{\eta}\left(m_{\rho}\right)=0.006 \mathrm{MeV}\right)$ and concentrate on the kaonic Casimir energy $\bar{E}_{\text {cas }}^{K}$ only.

As before we consider first the physical value for the kaon mass. Compared to $E_{\text {cas }}^{K}$ in the $S U(3)$ renormalization scheme the scale-dependence has reduced appreciably (Fig. 1, full line) indicating that indeed the $S U(2)$ renormalization scheme is more appropriate for the BSA. At scale $\mu=m_{\rho}$ we obtain $\bar{E}_{\text {cas }}^{K}\left(m_{\rho}\right)=-140 \mathrm{MeV}$ which added to the $S U(2)$ value of $1020 \mathrm{MeV}$ yields an acceptable estimate for the nucleon mass. As is noticed from Fig. 2 (full line) the dependence on the kaon mass seems to be reasonable, as expected i.e. the kaonic Casimir energy tends to zero with increasing kaon mass.

\section{DISCUSSION AND CONCLUSIONS}

We have studied the one-loop corrections to the $S U(3)$ skyrmion mass within the bound state soliton model. This approach assumes that along the strangeness direction only small amplitude fluctuations are possible. Thus, it is expected to become more accurate as the $S U(3)$ symmetry breaking terms increase. We found that the standard $S U(3)$ renormalization scheme leads to a considerable scale-dependence of the correction. Therefore, we introduced an alternative $S U(2)$ renormalization scheme. Such scheme yields an estimate of the total skyrmion mass which is in reasonable agreement with the empirical nucleon mass. Of course, this procedure is exact only in the limit $m_{K}=\infty$. To choose $m_{K}$ large but finite is an approximation since as soon as $m_{K}$ is finite (i) the classical solution should start to explore the strange sector and (ii) the $S U(2)$ renormalization scheme will be modified. Nevertheless, the BSA may be a good approximation for sufficiently large $m_{K}$. Actually, one could argue that this method might be trusted down to the region where the solid curve in Fig.2 has its minimum. For smaller $m_{K}$ the kaonic Casimir energy increases again although we know that the flavor symmetric value (dot in Fig. 3) lies much lower. Still, if one makes a smooth interpolation from the minimum up to the flavor symmetric point the resulting kaonic contribution does not differ very much from the value quoted above, $\bar{E}_{c a s}^{m_{K}}\left(m_{\rho}\right)=-140 \mathrm{MeV}$. In any case, what seems to be clear from Fig.2 is that the standard $S U(3)$ scheme together with BSA cannot be used to perform such interpolation. The slow rotator approach [11] appears as much well suited for that. However already in that approach the eta and kaonic components which are driven by the isospin breaking are missing. Thus, not even the slope of the Casimir energy at $m_{K}=m_{\pi}$ can be easily exactly calculated. Nevertheless, the simpler rigid rotator approach may still be a good approximation for sufficiently small $m_{K}>m_{\pi}$. Therefore, it would be interesting to complement the results of the present work with a calculation of the Casimir energy within the rigid rotator approach. There is even a possibility to obtain a smooth transition from small to large kaon masses in that way.

N.N.S. is fellow of the CONICET, Argentina. He also acknowledges a grant of the Fundación Antorchas, Argentina. H.W. is supported by a grant of JNICT, Portugal (Contract PRAXIS/2/2.1/FIS/451/94).

\section{Note added:}

Having finished this manuscript we became aware of a recently published paper by Kim and Park [12], which also reports on the kaonic Casimir energy. Using Moussallam's formula and neglecting the counter terms, their approach essentially corresponds to the standard $S U(3)$ regularization scheme discussed in Section 3.1. The difference of their number $+100 \mathrm{MeV}$ versus our $+140 \mathrm{MeV}$ for that case is mainly because we included all $\mathrm{ChO} 4$ terms in our lagrangian, most prominantly the kinetic symmetry breaker which influences the bound-state energies considerably. However, from our Fig.2 it becomes obvious that the kaonic Casimir energy should be negative in order to interpolate between $S U(2)$ and the $S U(3)$ flavor symmetric result. For that reason we considered the $S U(2)$ renormalization scheme (Section 3.2) more appropriate. This choice was supported by the moderate scale-dependence found in that case (Fig.1). 
[1] B. Moussallam and D. Kalafatis, Phys. Lett. B272, 196 (1991); B. Moussallam, Ann. Phys. 225, 264 (1993).

[2] F. Meier and H. Walliser, Phys.Rep. 289, 383 (1997).

[3] H. Yabu and K. Ando, Nucl. Phys. B301, 601 (1988).

[4] C.G. Callan and I. Klebanov, Nucl. Phys. B262, 365 (1985).

[5] N.N. Scoccola, H. Nadeau, M. Nowak and M. Rho, Phys. Lett. B201, 425 (1988); C.G. Callan, K Hornbostel and I. Klebanov, Phys. Lett. B202, 269 (1988); U. Blom, K. Dannbom and D.O. Riska, Nucl. Phys. A493, 384 (1989).

[6] H. Walliser, hep-ph/9710232 (Phys. Lett. B, in press).

[7] J. Gasser and H. Leutwyler, Nucl. Phys. B250, 465 (1985).

[8] G. Ecker, Czech. J. of Phys. 44, 405 (1995).

[9] C. Schat, N.N. Scoccola and C. Gobbi, Nucl. Phys. A585, 627 (1995).

[10] J. Gasser and H. Leutwyler, Ann. Phys. 158, 142 (1984).

[11] B. Schwesinger and H. Weigel, Nucl. Phys. A540, 461 (1992).

[12] J.-I. Kim and B.-Y. Park, Phys. Rev. D57, 2853 (1998).

TABLE I. Relation between the LECs $\bar{L}_{i}$ and the standard LECs $L_{i}$ together with the corresponding SU(2) and $S U(3)$ coefficients $\bar{\Gamma}_{i}$ and $\Gamma_{i} ; \nu_{K}$ and $\nu_{\eta}$ are defined in Eq.(17).

\begin{tabular}{|lrl|}
\hline \hline \hline $\bar{L}_{1}=L_{1}-\frac{1}{96} \nu_{K}$ & $\Gamma_{1}=\frac{3}{32}$ & $\bar{\Gamma}_{1}=\frac{1}{12}$ \\
$\bar{L}_{2}=L_{2}-\frac{1}{48} \nu_{K}$ & $\Gamma_{2}=\frac{3}{16}$ & $\bar{\Gamma}_{2}=\frac{1}{6}$ \\
$\bar{L}_{3}=L_{3}$ & $\Gamma_{3}=0$ & $\bar{\Gamma}_{3}=0$ \\
$\bar{L}_{4}=L_{4}$ & $\Gamma_{4}=\frac{1}{8}$ & $\bar{\Gamma}_{4}=\frac{1}{8}$ \\
$\bar{L}_{5}=L_{5}-\frac{1}{8} \nu_{K}$ & $\Gamma_{1}=\frac{3}{8}$ & $\bar{\Gamma}_{5}=\frac{1}{4}$ \\
$\bar{L}_{6}=L_{6}+\frac{1}{96} \nu_{K}+\frac{1}{144} \nu_{\eta}$ & $\Gamma_{6}=\frac{11}{144}$ & $\bar{\Gamma}_{6}=\frac{3}{32}$ \\
$\bar{L}_{7}=L_{7}$ & $\Gamma_{7}=0$ & $\bar{\Gamma}_{7}=0$ \\
$\bar{L}_{8}=L_{8}-\frac{1}{12} \nu_{K}-\frac{1}{48} \nu_{\eta}$ & $\Gamma_{8}=\frac{5}{48}$ & $\bar{\Gamma}_{8}=0$
\end{tabular}




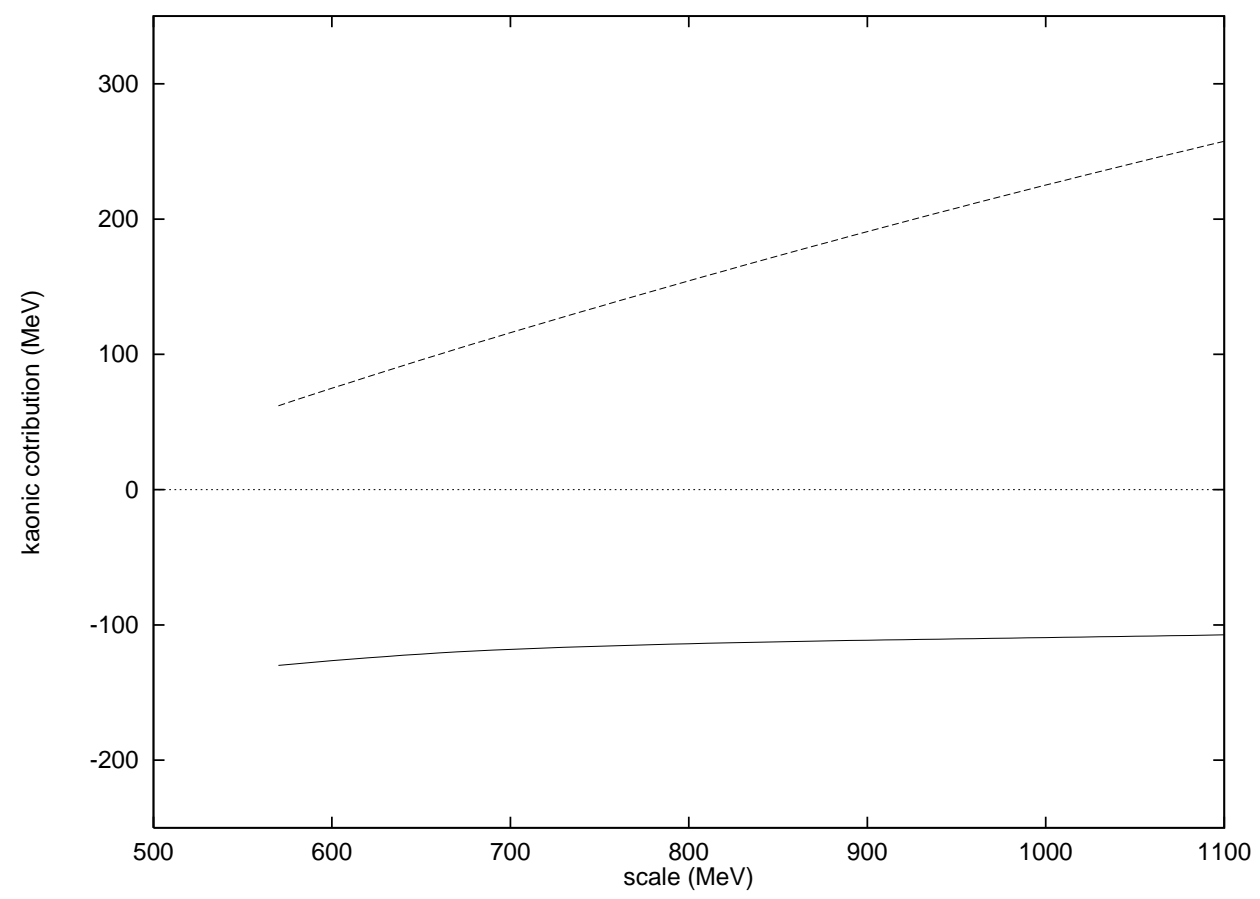

FIG. 1. Scale dependence of the Casimir corrections for empirical meson masses. Dashed line corresponds to the $S U(3)$ renormalization scheme whereas the full line corresponds to the $S U(2)$ scheme.

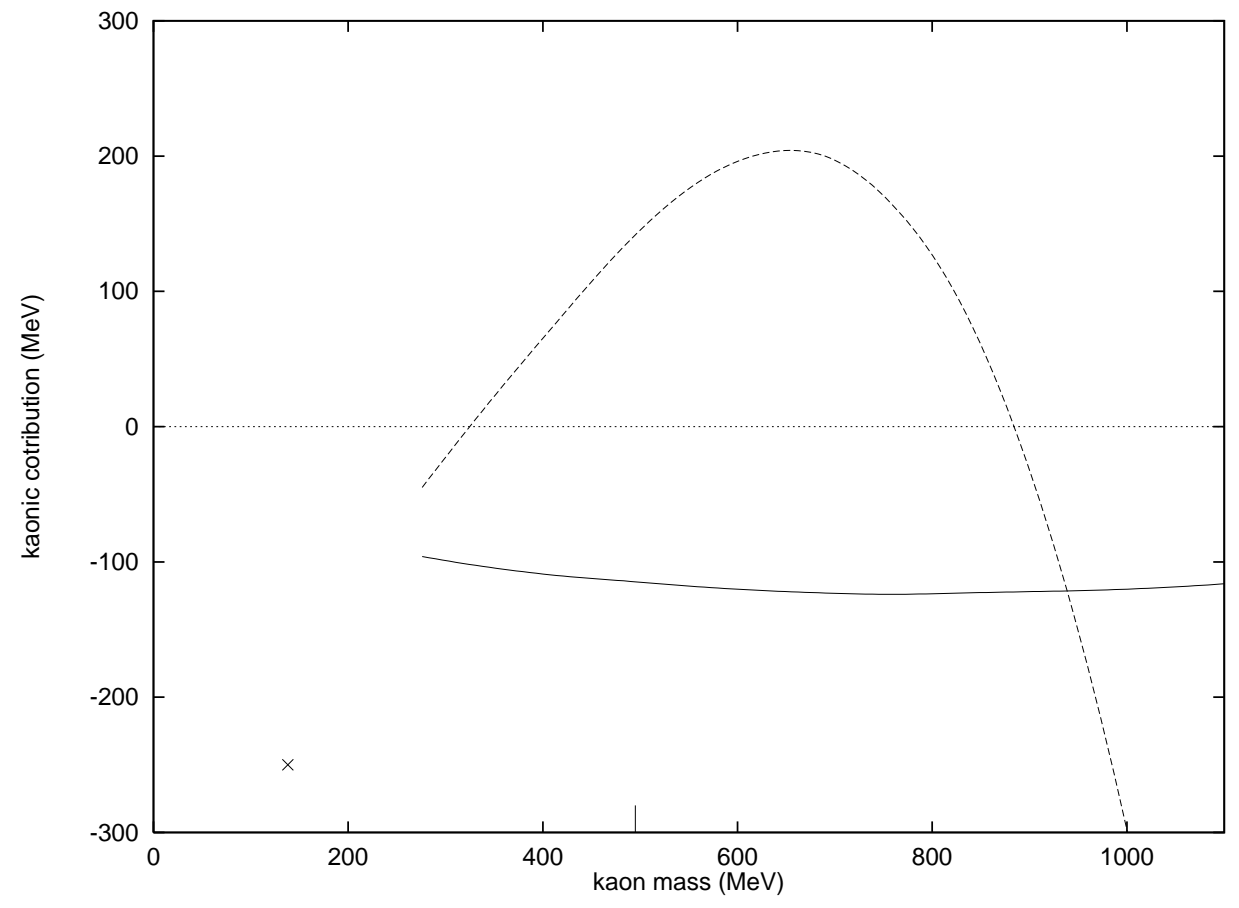

FIG. 2. Kaon mass dependence of the Casimir corrections. Dashed line corresponds to the SU(3) renormalization scheme whereas the full line corresponds to the $S U(2)$ scheme. The cross represents the flavor symmetric limit considered in Ref. [6]. The tick at $495 \mathrm{MeV}$ indicates the empirical kaon mass. 